\title{
Preparation and Properties of Starch-Based Colloidal Microgels
}

\author{
Yannick Dziechciarek, ${ }^{*} \dagger$ Jeroen J. G. van Soest, ${ }^{*}$ and Albert P. Philipse ${ }^{,} 1$ \\ * Agrotechnological Research Institute (ATO), P.O. Box 17, 6700 AA, Wageningen, The Netherlands; and †Van't Hoff Laboratory for Physical \\ and Colloid Chemistry, Debye Institute, Utrecht University, Padualaan 8, 3524 CH Utrecht, The Netherlands
}

Received April 2, 2001; accepted October 17, 2001

\begin{abstract}
Novel starch microgels were prepared by emulsion cross-linking and characterized with respect to shape, volume, and mass density. Starch microgels appear to be negatively charged $(\sim-50 \mathrm{mV})$, with a particle size varying as a function of the type of cross-linker (ca. 0.25-10 $\mu \mathrm{m}$ ). Environmental scanning electron microscopy observations show a dependence of the particle swelling on the cross-linking density. Viscosimetry reveals that starch microgels behave as charged polymers, where the reduced viscosity increases with dilution (anomalous viscosity behavior) for sufficiently low $\kappa a$ (ca. $\kappa a<3$ ), the ratio of the particle radius $(a)$, and the Debye length $\left(\kappa^{-1}\right)$. Analogous results are obtained for reference-charged rigid silica spheres, which approach the hard sphere limit for increasing ionic strengths. The shape of the microgels appears to play a minor role in the anomalous viscosity behavior, which is more likely dominated by electrostatic effects. $\mathcal{O} 2002$ Elsevier Science

Key Words: starch microgels; charged silica spheres; environmental scanning electron microscopy; ionic strength; intrinsic viscosity, starch colloids.
\end{abstract}

\section{INTRODUCTION}

Starch is an inexpensive and abundant polysaccharide, which is found in nature as water-insoluble semi-crystalline granules with sizes varying from 0.5 to $100 \mu \mathrm{m}$. Although starch is easily gelatinized or dissolved in water, it is still difficult to process due to its high viscosity and shear thickening properties, which already occur at low solid contents. The range of functional properties of starch is restricted even for chemically modified starch with a high degree of substitution $(1,2)$. As a consequence of these limitations, it is not possible to form stable suspensions or to obtain colloidal systems from native starches. This is a disadvantage of starch for potential applications, such as, papermaking, ink, coatings, and bio-pharmaceutical products (3).

Recently, novel starch-based microgels were prepared, which behave as colloids in aqueous suspension (4). The synthesis is based on a combination of gelatinization and cross-linking performed in water-in-oil emulsions. The hydroxyl groups of the repeating glucose units of the starch chains, or other groups intro-

\footnotetext{
${ }^{1}$ To whom correspondence should be addressed. Fax: ++31-30-2533870. E-mail: FCCoffice@chem.uu.nl.
}

duced chemically, react with a cross-linking agent in an inverse emulsion $(4,5)$. An extensive list of cross-linkers is known for starch, and phosphoryl chloride, epichlorohydrin, and tri-sodium tri-metaphosphate are the most common. Their cross-linking reactions are reported elsewhere (6-8). Starch-based submicrogels are biodegradable and may be used in both food and nonfood applications like thickeners or emulsifiers in paints and coatings.

In most applications, volume and conformation changes of starch microgels are crucial since they determine, for example, the rheology of suspensions (9-11). Shape, size, and structure of starch colloids, as well as their specific volume and swelling behavior, have had little study, thus far. The aim of this research is, therefore, not only to establish synthesis procedures for starch colloids but also to investigate single-particle properties such as size and swelling.

Starch microgels will be characterized using X-ray diffraction, optical and electron microscopy, light scattering, microelectrophoresis, and densitometry. New developments in the field of electron microscopy allowed the opportunity to study the swelling of starch colloids with environmental scanning electron microscopy (E-SEM) $(12,13)$. Contrary to conventional electron microscopy, the relative moisture content in the E-SEM sample chamber can be varied easily, inducing possible deformations of starch microgels. This novel technique might be relevant for a fast determination of the swelling degree of hydrophilic materials or for investigations of the dynamics of samples containing volatile components.

In the literature on the rheology of polymer colloids, particlespecific volumes are determined via intrinsic viscosity measurements, modeling the particles as noninteracting spheres (9-11). However, for polyelectrolytes, the reduced viscosity is known to increase with dilution at low ionic strengths (14-17). This anomalous viscosity behavior is explained in terms of changes of the polymer shape (14-16), or more recently, in terms of electrostatic effects (17). In this paper, reduced viscosity measurements were performed to determine whether starch microgels behave like charged polymers in aqueous suspensions. In the affirmative case, as native starch granules tend to swell in aqueous suspensions (18), the viscosimetric results obtained for starch microgels will be compared with the rheology of charged stabilized rigid silica spheres at low particle concentrations and for increasing ionic strengths. This will 
allow verification of which contribution (i.e., change of shape or electrostatics) has the major effect on the anomalous viscosity behavior. The silica spheres are monodisperse in size, undeformable, and well-characterized (19) and present convenient reference particles for the more complicated starch-based microgels.

\section{EXPERIMENTAL}

\subsection{Materials}

Paselli SA-2, an enzymatically modified potato starch, was supplied by Avebe (moisture content $\sim 10 \%$ w/w). Analytical grade epichlorohydrin $(\mathrm{ECH})$, trisodium trimetaphosphate (TSTP), and cyclohexane were supplied by Merck. Sorbitan mono-oleate (Span 80, Boom) was used as the emulsifier and acetic acid (99\%, Boom) as the emulsion breaking agent. The charge-stabilized silica particles were deionized using mixed bed resins (AG sol-X (8) D) supplied by Biorad.

\subsection{Preparation of the Starch Colloids}

An illustrative and reproducible preparation procedure of the starch microgels will be described in this section. The composition of all stock dispersions is shown in Table 1. For example, sample 20Pa1E1O is coded as 20PaSA2/1ECH/1OH, which expresses the amount (in grams) of the individual components in $100 \mathrm{~g}$ water used to prepare the hydrophilic phase. Initially, $20 \mathrm{~g}$ of starch was dispersed in a beaker containing $100 \mathrm{~g}$ of a $0.25 \mathrm{M} \mathrm{NaOH}$ aqueous solution. The suspension was stirred at $800 \mathrm{rpm}$ at $50^{\circ} \mathrm{C}$ until all starch was dissolved. Subsequently, $1.7 \mathrm{ml} \mathrm{ECH}$ was added to the mixture. After complete mixing, the hydrophilic phase was poured gradually into a two-neck round bottom flask containing a solution of $5 \mathrm{~g}$ emulsifier dissolved in $250 \mathrm{ml}$ of cyclohexane. The resulting white emulsion was stirred with an Ultra-Turrax stirrer type T125 at $24.000 \mathrm{rpm}$ for $10 \mathrm{~min}$. The temperature was kept between 35 and $45^{\circ} \mathrm{C}$. The cross-linking reaction took place at room temperature and under constant stirring speed conditions (magnetic stirrer, $800 \mathrm{rpm}$ ).

TABLE 1

List of the Stock Dispersions and Their Composition ${ }^{a}$

\begin{tabular}{ll}
\hline Stock dispersions & \multicolumn{1}{c}{ Compositions } \\
\hline 20Pa1E1O & 20 Paselli SA-2/1ECH/1OH \\
20Pa2E1O & 20 Paselli SA-2/2ECH/1OH \\
20Pa4.9E1O & 20 Paselli SA-2/4.9ECH/1OH \\
40Pa2E1O & 40 Paselli SA-2/2ECH/1OH \\
20Pa4T1OA & 20 Paselli SA-2/4TSTP/1OH \\
20Pa4T1OB & 20 Paselli SA-2/4TSTP/1OH \\
\hline
\end{tabular}

\footnotetext{
${ }^{a}$ The composition of the stock dispersions is expressed in grams of the individual components per $100 \mathrm{~g}$ water, used to form the hydrophilic phase during the formation of the emulsion. For example, the composition of 20Pa1E1O was labeled 20 Paselli SA-2/1ECH/1OH, which refers to $20 \mathrm{~g}$ Paselli SA-2, $1 \mathrm{~g}$ epichlorohydrin, and $1 \mathrm{~g} \mathrm{NaOH}$ dispersed in $100 \mathrm{~g}$ demineralized water.
}

TABLE 2

Radius Combined with Zeta-Potential and Mass Particle Density of Salt-Free Starch-Based and Silica Particles at $20.00^{\circ} \mathrm{C}$

\begin{tabular}{lcccc}
\hline \multicolumn{1}{c}{ Sample } & $a(\mathrm{~nm})^{a}$ & $\sigma(\%)$ & $\begin{array}{c}\text { Zeta-potential }(\mathrm{mV}) \\
(+/-2.5 \mathrm{mV})\end{array}$ & $\rho_{\text {part }}(\mathrm{g} / \mathrm{ml})$ \\
\hline 20Pa1E1O & 128 & 30 & -45 & 1.70 \\
20Pa2E1O & 192 & 52 & -42 & 1.65 \\
20Pa4.9E1O & 178 & 39 & -45 & 1.36 \\
20Pa4T1OA & $2500^{b}$ & - & -50 & 1.70 \\
20Pa4T1OB & 304 & 24 & -50 & 1.70 \\
DB373 & 145 & 2 & -40 & 1.60 \\
\hline
\end{tabular}

${ }^{a}$ Determined by static light scattering.

${ }^{b}$ Determined by optical microscopy.

After $20 \mathrm{~h}$ of reaction, the emulsion was transferred to a separation funnel, where $250 \mathrm{ml}$ of acetic acid was added to separate the hydrophilic phase containing the starch microgels. The resulting aqueous suspension was purified from the residual salts by repetitive washings with demineralized water and ethanol by means of a Beckman centrifuge (30 min at 10,000 rpm). The particles were dialyzed at $4{ }^{\circ} \mathrm{C}$ against freshly demineralized water until the conductivity of the aqueous suspensions, called stock dispersions, was less than $0.1 \mathrm{mS} / \mathrm{cm}$. The resulting $\mathrm{pH}$ of the stock dispersion was about 5-6. To prevent any fungal and bacterial growth in the suspensions, the particle dispersions were stored at $4^{\circ} \mathrm{C}$.

\subsection{Charge-Stabilized Silica Particles}

TPM-silica spheres are used in this study as reference materials for the rheology experiments. Size, zeta-potential, and mass density of the colloids are reported in Table 2 . The synthesis of charge-stabilized TPM-silica spheres (labeled DB373) in ethanol has been described in detail elsewhere (19). The particles are coated with 3-methacryloxypropyltrimethoxysilane, denoted as TPM. For the deionized suspensions, mixed bead resins (5 g/L) were added to the dilutions. The samples had been deionizing for at least 5 days in a Teflon bottle prior to use.

\subsection{Preparation of Suspensions for Rheological Measurements}

In order to determine the influence of the particle concentration on the viscosity of dilute dispersions of starch microgels and TPM-silica particles, the weight concentration of the milky stock suspension was determined by drying a fraction to constant weight under an infrared lamp. A series of suspensions were prepared by diluting the stock suspension with demineralized water and $\mathrm{NaCl}$ solutions for the starch microgels, and absolute ethanol and $\mathrm{LiNO}_{3}$ solutions for the TPM-silica particles. All suspensions had a transparent bluish color and no sedimentation was observed for more than 3 months. 


\subsection{Calculation of the Debye Length}

As the rheological behavior of several suspensions of starch microgels and charged silica spheres will be investigated with variations in the ionic strength, the Debye length $\left(\kappa^{-1}\right)$ is a suitable parameter for the comparison of the results. Assuming monovalent counter- and salt ions in the suspensions (20), $\kappa^{-1}$ is given by

$$
\kappa^{2}=\left(\kappa_{\mathrm{c}}\right)^{2}+\left(\kappa_{\mathrm{s}}\right)^{2}=4 \pi L_{\mathrm{B}} n\left|Z^{\mathrm{eff}}\right|+8 \pi L_{\mathrm{B}} N_{\mathrm{Av}} c_{\mathrm{s}} .
$$

Here $\kappa_{\mathrm{c}}$ stems from the contribution of the counterions produced by the particles themselves, while $\kappa_{\mathrm{s}}$ is due to the external salt concentration, $n$ is the number density of colloids, $Z^{\text {eff }}$ the effective colloid valency, $N_{\mathrm{Av}}$ Avogadro's number, and $c_{\mathrm{s}}$ the salt concentration. $L_{\mathrm{B}}$ is the Bjerrum length:

$$
L_{\mathrm{B}}=\frac{e^{2}}{4 \pi \varepsilon \varepsilon_{0} k_{\mathrm{B}} T},
$$

where $\varepsilon$ is the dielectric constant of the solvent, $\varepsilon_{0}$ the permittivity of the vacuum, and $e$ the elementary charge. Since counterions densities are unknown, we calculated $\kappa_{\mathrm{s}}^{-1}$ for starch microgels in water and for TPM-silica particles in absolute ethanol (see Table 3) from

$$
\kappa_{\mathrm{s}}^{2}=8 \pi L_{\mathrm{B}} N_{\mathrm{Av}} c_{\mathrm{s}}
$$

For salt-free dispersions, $c_{\mathrm{s}}$ was estimated from the conductivity of the solvent, assuming that charge carriers in water and in ethanol are monovalent (20). For deionized systems, $\kappa^{-1}$ could not be determined since the conductivity was too low to be measurable. For such systems, $\kappa_{\mathrm{s}}^{-1}$ can be estimated from the average interparticle distance in the final sediment of concentrated suspensions at equilibrium, to be about 1.5-2 times the Debye

TABLE 3

Electrolyte Concentrations and Debye Lengths in Aqueous Sus-

\begin{tabular}{|c|c|c|}
\hline (a) & $\mathrm{c}_{\mathrm{NaCl}}\left(\mathrm{mol} / \mathrm{m}^{-3}\right)$ & $\kappa_{\mathrm{s}}^{-1}(\mathrm{~nm})$ \\
\hline & 0.01 & 95 \\
\hline & 0.06 & 40 \\
\hline & 3.4 & 5 \\
\hline & 15 & 2.5 \\
\hline & 1000 & 0 \\
\hline \multirow[t]{4}{*}{ (b) } & $\mathrm{c}_{\mathrm{LiNO} 3}\left(\mathrm{~mol} / \mathrm{m}^{-3}\right)$ & $\kappa_{\mathrm{s}}^{-1}(\mathrm{~nm})$ \\
\hline & $\complement^{a}$ & Not measurable \\
\hline & $0.005^{b}$ & 75 \\
\hline & 0.24 & 9 \\
\hline
\end{tabular}
pensions for Starch-Based Particles (a) and in Suspensions for Silica Particles in Ethanol (b)

\footnotetext{
${ }^{a}$ Deionized system.

${ }^{b}$ No salt added.
}

length $\kappa^{-1}$ (21). For TPM-silica particles in deionized ethanol, $\kappa^{-1}$ was estimated to be in the range of $200-400 \mathrm{~nm}$.

\subsection{Characterization Techniques}

$\mathrm{X}$-ray diffraction is a proven method to determine and eventually quantify crystal patterns in the starch microgels (22). Crystal impurities remaining in the prepared samples can be detected as well. X-ray experiments were carried out on dried starch-based particles with a Philips PC-APD diffractometer (Model PW370) equipped with a Cu-X-ray tube $\left(\alpha_{\lambda}=1.542 \AA\right)$. The power was set on $2.0 \mathrm{~kW}(40 \mathrm{kV}, 50 \mathrm{~mA})$. The detector, adapted with a Nickel filter, rotates with an angle of 4 to $40^{\circ}(2 \theta)$, in 720 steps of $2 \mathrm{~s}$.

Time-averaged light scattering intensities were measured in a Fica-50 light scattering photometer using vertically polarized incident and detected light $(\lambda=546 \mathrm{~nm})$. The scattering angle was varied between $\theta=30^{\circ}$ and $\theta=150^{\circ}$. The samples were filtered into the measuring cell through a Millipore filter (pore diameter $=10 \mu \mathrm{m}$ ). All measurements were performed at $25.0^{\circ} \mathrm{C}$. From the radius of gyration a particle radius was estimated by using a Guinier plot, where $a=(5 / 3)^{1 / 2} R_{\mathrm{g}}\left(R_{\mathrm{g}}\right.$ being the radius of gyration of the particle, which is assumed to be a sphere) (10). Particles larger than $1 \mu \mathrm{m}$ were observed with a Zeiss Axioplan MC1000 light microscope equipped with a scaling bar. The average particle diameter and the size distribution were estimated by measuring the diameter of at least 50 starch microgels.

The mobility of starch-based particles in salt-free suspensions was measured with a Coulter Delsa 440 SX electrophoresis meter. The Smoluchowski approximation was used, assuming that the ratio of the particle radius and the Debye length $(\kappa a)$ was $\gg 1$ for all suspensions (10),

$$
u_{\mathrm{E}}=\frac{\varepsilon \varepsilon_{0} \zeta}{\eta}
$$

where $u_{\mathrm{E}}$ is the electrophoretic mobility $\left(\mathrm{m}^{2} / \mathrm{V} \cdot \mathrm{s}\right), \eta$ the viscosity of the solvent, and $\zeta$ is the zeta-potential $(\mathrm{mV})$.

Particle mass densities were determined with a DA 200 high precision density meter. A thermostated U-tube filled with $1.5 \mathrm{ml}$ of dispersion oscillated harmonically with fixed amplitude. With the dispersion density $\left(\rho_{\text {disp }}\right)$ being proportional to the resonance period (23), the particle density $\left(\rho_{\text {part }}\right)$ was determined from the dispersion density and the solvent density $\left(\rho_{\text {solv }}\right)$ according to

$$
\frac{d \rho_{\text {disp }}}{d c_{\mathrm{p}}}=1-\frac{\rho_{\text {solv }}}{\rho_{\text {part }}}
$$

Here $c_{\mathrm{p}}$ is the weight concentration of the particles in the dispersions. All density measurements were performed at $20.00^{\circ} \mathrm{C}$.

Starch microgels were observed with a JEOL 5600 LV SEM and a Philips XL 30 E-SEM-LaB6. Normally, samples for conventional scanning electron microscopy must be clean, dry, vacuum compatible, and electrically conductive. This is not the case 
for starch microgels, which are very hydrophilic, nonconductive, and sensitive to electron bombardment. Still, starch microgels can be examined if they are sufficiently dried (less than $15 \%$ relative moisture content) and coated with a thin conducting layer of gold, giving a suitable yield of secondary electrons and, thus, a good image quality. However, this treatment prevents the observation of any conformation changes of starch colloids in a relatively high-moistened environment. Since the elaboration of the gaseous secondary electron detector (GSED) (12), a novel type of SEM (environmental SEM) (13) has been developed allowing the observation of samples in an environment, within which relative moisture content can be varied. A highly moistened sample is placed in an environmental chamber isolated from the main SEM column by one or more differential pumps. The secondary electron signal is enhanced by cascade amplification and is detected by the GSED. This cascade amplification also produces positive ions attracted by negative charges on the sample suppressing charging artefacts. The prepared starchbased samples were observed at $5^{\circ} \mathrm{C}$ and at increasing vapor pressures gradually from 2.9 up to 6 Torr, corresponding respectively to 20 and $100 \%$ relative moisture content in the sample chamber. TPM-silica particles were observed with transmission electron miscoscopy. A small carbon grid was immersed in the stock dispersion of TPM-silica particles, air-dried, and then finally placed in the TEM chamber. The estimation of the particle radius was performed on the average of about 70 particles for each sample.

Low-shear viscosity experiments were carried out with a constant shear Contraves LS 40 rheometer in an MS 41/1S Couette geometry. The temperature of the geometry was kept constant at $20.0^{\circ} \mathrm{C}\left( \pm 0.1^{\circ} \mathrm{C}\right)$, by the circulation of water from a thermostatic bath to the measuring device. Using a Finn pipette, $2 \mathrm{ml}$ of the sample was introduced in the geometry. The measurements were performed in a shear rate range from $D=0.1 \mathrm{~s}^{-1}$ up to $D=10 \mathrm{~s}^{-1}$. In every run, the shear stress was measured for 15 different shear rates, evenly spaced. This range and the dilutions of the stock dispersions were chosen to remain within the lowshear Newtonian region. The stock dispersion was diluted in such a way that the viscosity difference of particle suspensions and solvent remained above $8 \%$. The flow curves were measured successively for increasing and decreasing shear rates and each shear rate was applied for $40 \mathrm{~s}$, after which the shear stress was measured. The slope yields the viscosity of the dispersions $\left(\eta_{\text {disp }}\right)$ and of the solvent $\left(\eta_{\text {solv }}\right)$, and thus the relative viscosity is defined as

$$
\eta_{\mathrm{r}}=\frac{\eta_{\text {disp }}}{\eta_{\text {solv }}}
$$

According to Einstein's equation for hard spheres,

$$
\eta_{\mathrm{sp}}=\eta_{\mathrm{r}}-1=2.5 \phi
$$

where $\eta_{\mathrm{sp}}$ is the specific viscosity and $\phi$ is the particle volume fraction, the reduced viscosity $\left(\eta_{\mathrm{red}}=\eta_{\mathrm{sp}} / c_{\mathrm{p}}\right)$ can be expressed in terms of molecular parameters (17):

$$
\lim _{c_{\mathrm{p}}, D \rightarrow 0} \eta_{\mathrm{red}} \equiv[\eta]=(10 / 3) \pi N_{\mathrm{A}}\left(R_{\eta}^{3} / M_{\mathrm{W}}\right) .
$$

Here, $M_{\mathrm{W}}$ is the particle molecular weight, $R_{\eta}$ is the radius at the shear plane, $c_{\mathrm{p}}$ is the colloid concentration (in $\mathrm{g} / \mathrm{ml}$ ). For higher concentrations of neutral polymers, the intrinsic viscosity $([\eta])$ and the Huggins coefficient $\left(k_{\mathrm{H}}\right)$ are usually determined by plotting the specific viscosity as a function of $c_{\mathrm{p}}$ (see, for example, (17)):

$$
\eta_{\text {red }}\left(c_{\mathrm{p}}\right)=[\eta]+k_{\mathrm{H}} \cdot[\eta]^{2} \cdot c_{\mathrm{p}}
$$

Here $[\eta]$ is expressed in $[\mathrm{ml} / \mathrm{g}]$.

\section{RESULTS AND DISCUSSION}

\subsection{Structure, Shape, Size, and Charge of Starch Microgels}

$\mathrm{X}$-ray diffraction was applied as starch granules present typical semicrystalline structures, composed of amylopectin (70 to $80 \%$ ) and amorphous amylose chains (20 to $30 \%$ ). Native potato starch exhibits a B-pattern structure, built out of parallel, double helixes of mainly amylopectin. A hexagonal packed cell of these helixes contains a hole in the center, filled with 36 bound water molecules. Typical X-ray diffraction peaks for the B pattern are $5,17,22$, and $24^{\circ}(2 \theta)(22)$. X-ray diffractograms of starch microgels did not show crystal peaks inherent to starch nor characteristic peaks of residual crystalline (poly)phosphates or acetate.

The starch microgels and silica spheres were characterized with regard to their shape and size. Figure 1a shows a SEM photograph of sample 20Pa2E1O: the microgels are spherical with a low polydispersity in size. The microgels are apparently very sensitive to the electron beam, as they were already melting rapidly during the time the samples were observed. Figure $1 \mathrm{~b}$ shows a TEM picture of TPM-silica spheres. Though the particles slightly shrink in vacuum (certainly less than $2 \%$ ), the particles are assumed to behave as rigid spheres in the rheology experiments (see section 3.3.).

Light scattering data, reported in Table 2, inform us that the size of starch microgels ranges from $180 \mathrm{~nm}$ up to $5 \mu \mathrm{m}$ with a large size distribution, compared to TPM-silica spheres DB373. The polydispersity of starch colloids is attributed to the method of preparation of the emulsion. To decrease the droplets size, the hydrophilic and hydrophobic phases had to be mixed in a short time at a high mechanical input. Such a high energy input per unit of time is responsible for the increase of the polydispersity in droplets size (24).

The size of ECH-cross-linked particles is about 10 times smaller than the colloids cross-linked with TSTP. Two explanations can be put forward. On one hand, during the preparation of starch colloids, the discrete phase containing ECH is easily dispersed in cyclohexane, since both densities are comparable. 


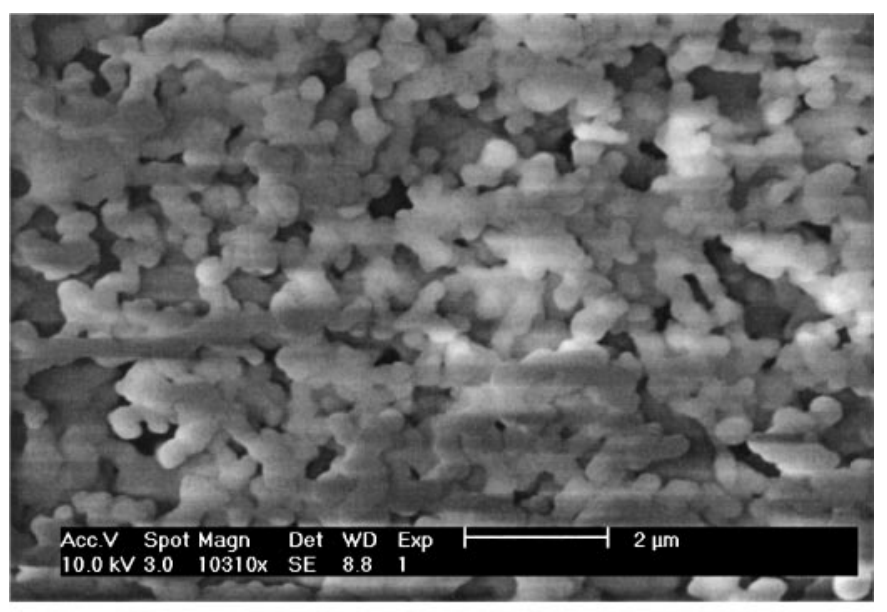

a)

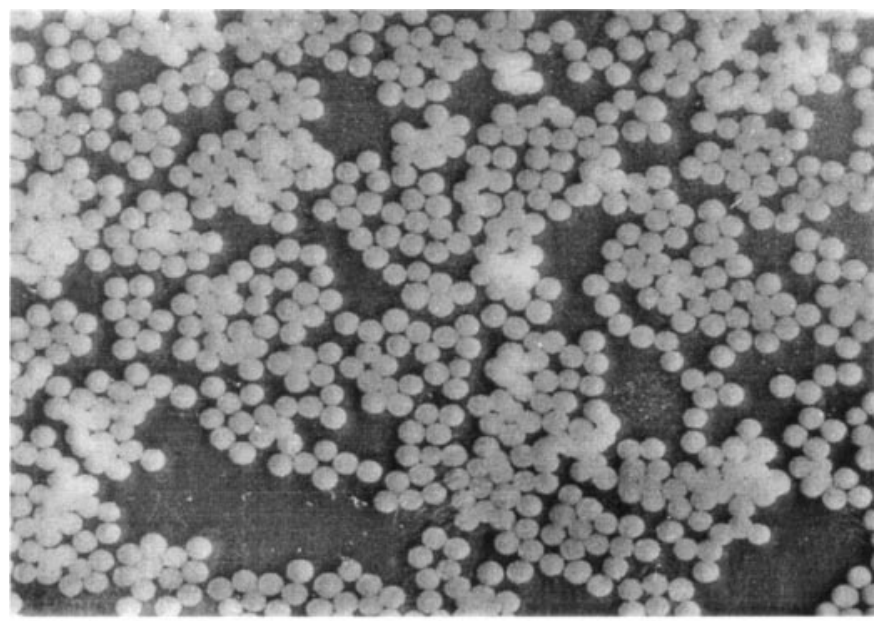

b)

FIG. 1. (a) SEM picture of sample 20Pa2E1OH in a dry state $(a=200 \mathrm{~nm})$; (b) TEM picture of TPM-silica particles (DB373) $(a=145 \mathrm{~nm})$.

However, the density of the TSTP hydrophilic phase is almost 10 times larger than the continuous phase (cyclohexane). This difference in densities leads to larger droplets, and consequently, to the formation of larger hydrogels (5). On the other hand, large internal electrostatic repulsions between phosphate diester cross-links are introduced during the synthesis of TSTP particles. These repulsions might be responsible for a larger swelling of the particles in aqueous suspension, in comparison with ECH colloids, containing glycerol-like cross-links (25). Higher electrophoretical mobilities for TSTP-microgels could be expected in view of their remarkable swelling.

Hence, to observe any influence of charge on the swelling, zeta-potentials of starch microgels were measured by microelectrophoresis; the results are reported in Table 2. Results show that starch microgels are negatively charged, which is most likely due to the large amount of dissociating alcohol groups of the glucose units (25). In addition, ECH-cross-linked microgels had zeta-potentials of the same magnitude as for TSTP-cross-linked

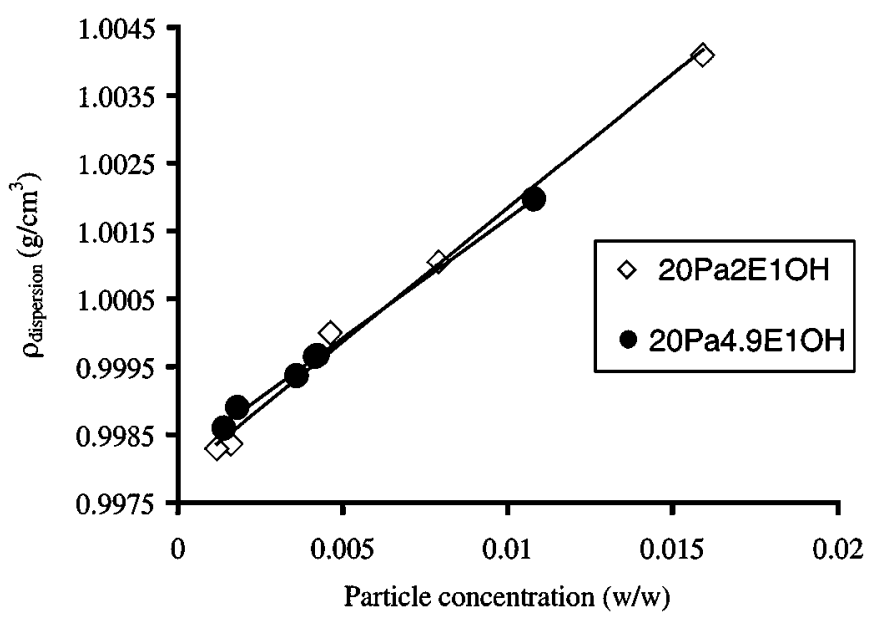

FIG. 2. Density of $(\bullet) 20 \mathrm{~Pa} 2 \mathrm{E} 1 \mathrm{OH}$ and $(\diamond) 20 \mathrm{~Pa} 4.9 \mathrm{E} 1 \mathrm{OH}$ suspensions versus the particle concentration, at $20.00^{\circ} \mathrm{C}$. The suspension densities are of the same magnitude as water. A linear fit of the data provides the dry particle density.

microgels. Modest differences in zeta-potentials are not large enough to be observed with electrophoretical mobility measurements. Hence, it is difficult with this characterization technique to demonstrate unambiguously the contribution of the charged phosphate cross-links to the swelling of the microgels. Note in Table 2 that the zeta-potential and the size of TPM-silica spheres are comparable with ECH-starch colloids, which makes the charged silica spheres suitable reference particles for the more complex starch microgels.

\subsection{Density and Swelling of Starch Colloids}

Particle densities were investigated by densitometry, and particle swelling was studied by E-SEM and viscosimetry. In Table 2, the dry particle mass densities obtained for starch colloids are similar to those of dry potato starches $(1.53-1.56 \mathrm{~g} / \mathrm{ml})$ (26). The mass density of silica spheres (DB373) is $1.6 \mathrm{~g} / \mathrm{ml}$ and is comparable to the mass density of the starch microgels. In Fig. 2, densities of dilute suspensions of the starch microgels

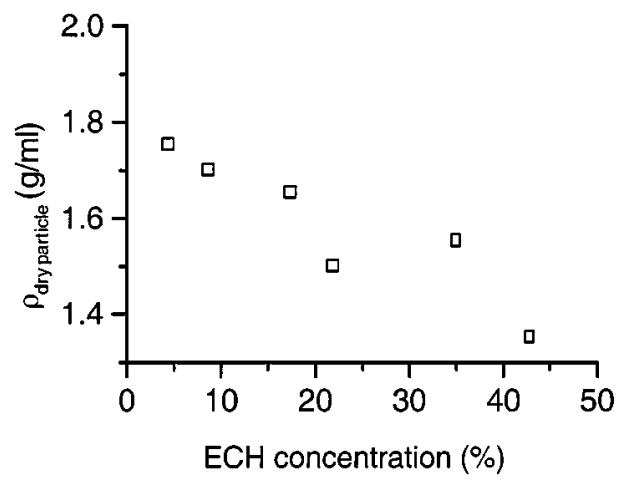

FIG. 3. Influence of the cross-linker concentration (ECH) on the particle dry density of starch-based microgels. The decrease of the density is probably due to a minor affinity of the low cross-linked particles for the aqueous suspension. 
are depicted as a function of the particle concentration. The measured curves are straight lines (with a correlation coefficient $r>0.995)$. The suspension densities are similar to water, implying that the particles are porous. In Fig. 3, the mass densities of starch colloids are plotted against the cross-linker concentration (epichlohydrin). Surprisingly, a decrease in mass density was observed as the amount of cross-linker was increased. Similar results, related to the swelling of sparsely cross-linked ethyl acetate cellulose gels, were reported earlier and were attributed to low affinity of the polymer with the solvent (27).

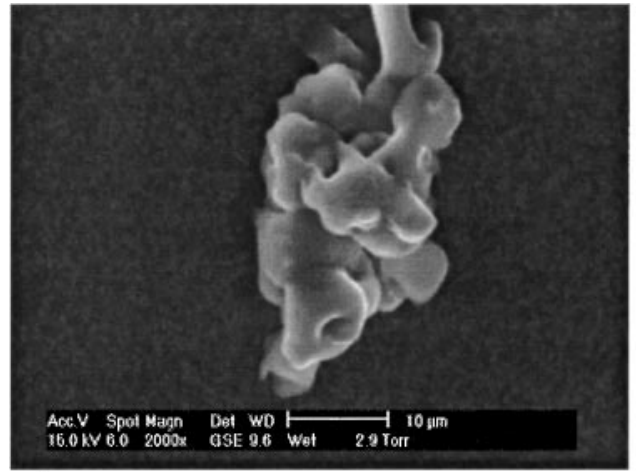

a)

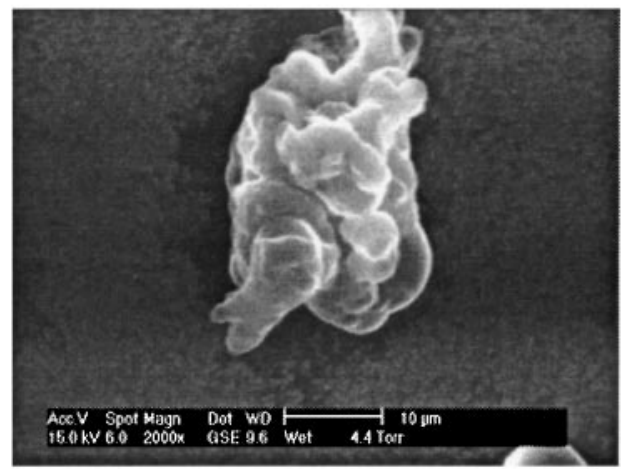

c)

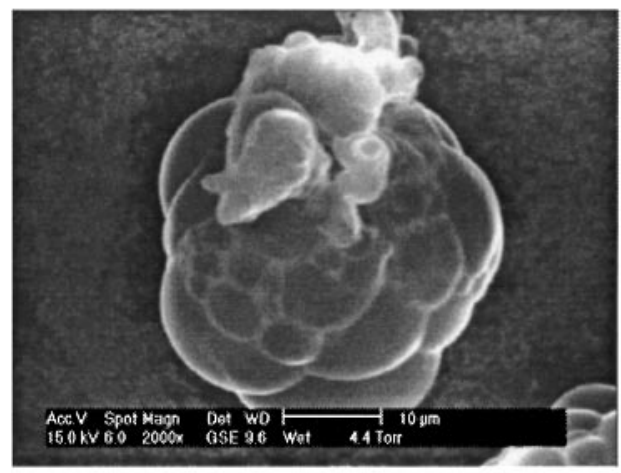

e)
The swelling of dry starch microgels was observed by E-SEM, by increasing the environmental moisture content. The swelling behavior of sample 20Pa1E1O is displayed in Fig. 4. At 20\% relative moisture content $(\mathrm{rmc})$, the particles become gel-like agglomerated, as already noted in section 3.1 . At $60 \% \mathrm{rmc}$, the aggregates start to swell, and at $70 \%$ the particles are swollen up to 10 times their initial volume. This high swelling is probably related to the low efficiency of the cross-linking reaction $(6,7)$. It was already shown that during the cross-linking reaction of epichlorohydrin with polysaccharides $(28,29)$, only a minor

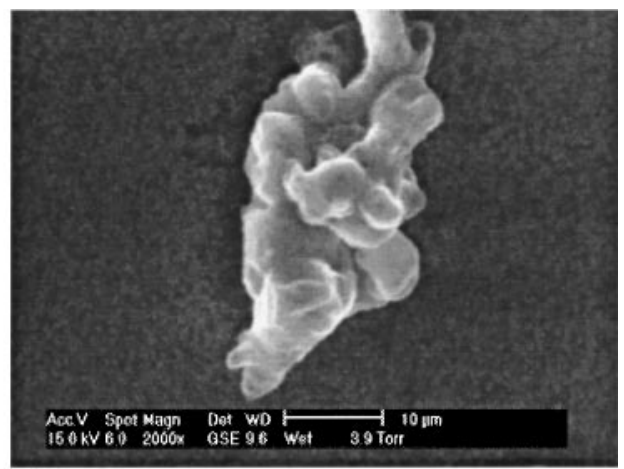

b)

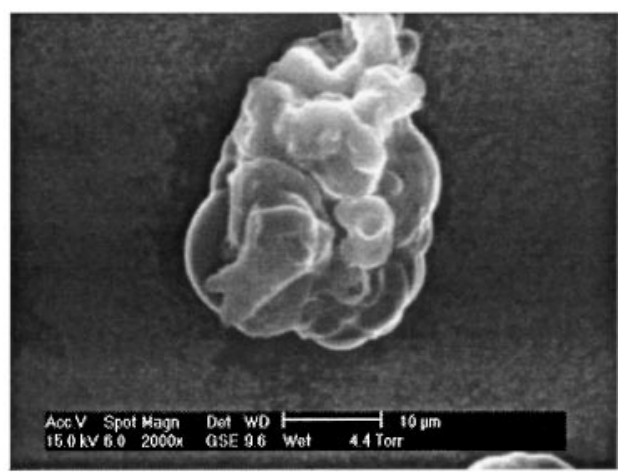

d)

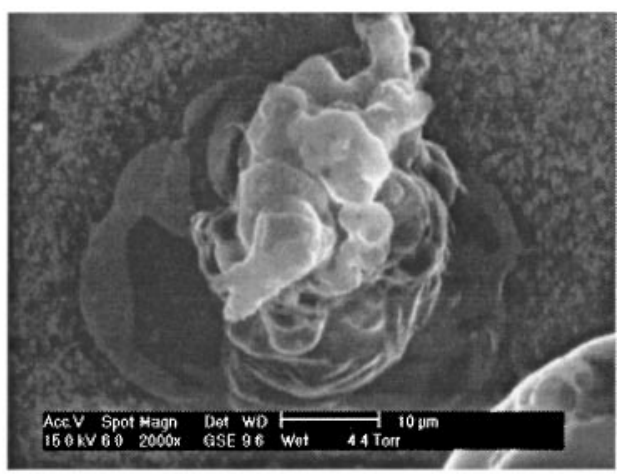

f)

FIG. 4. E-SEM pictures of sample $20 \mathrm{~Pa} 1 \mathrm{E} 1 \mathrm{OH}$ for (a) $20 \%$ (b) $60 \%$, and (c-f) $70 \%$ relative moisture content $\left(5^{\circ} \mathrm{C}\right)$. 


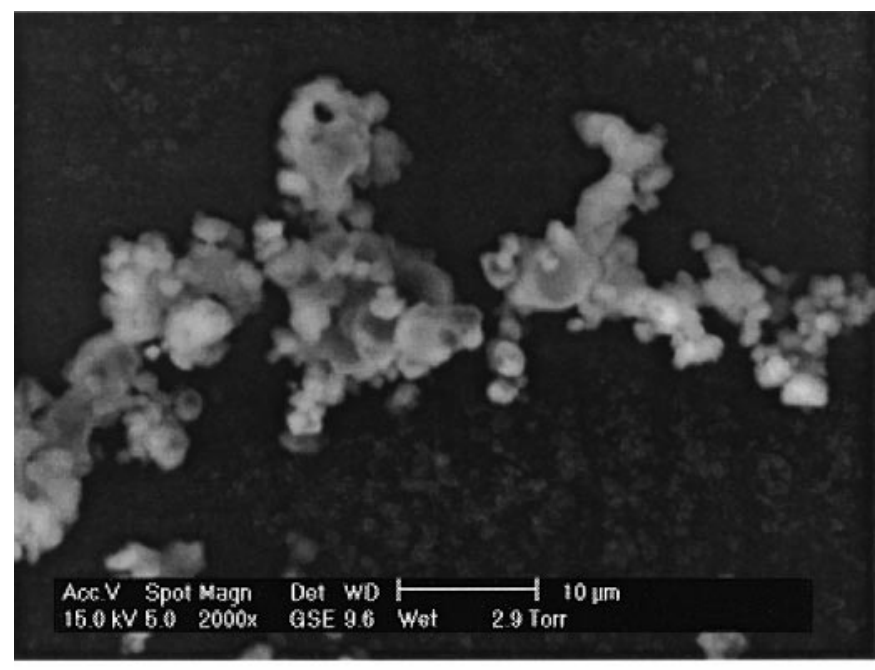

a)

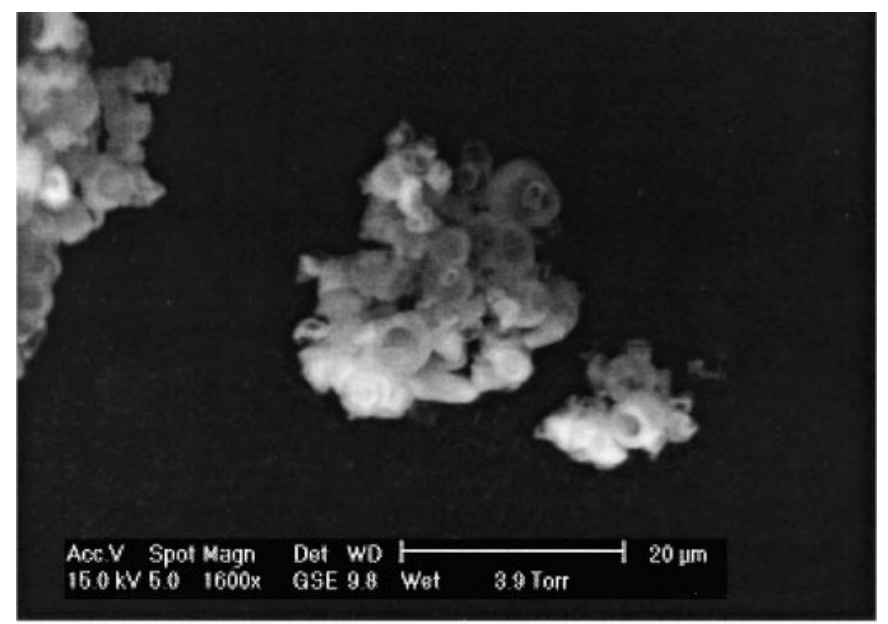

b)

FIG. 5. E-SEM picture of samples (a) 20Pa4.9E1OH and (b) $40 \mathrm{~Pa} 2 \mathrm{E} 1 \mathrm{OH}$ $\left(5^{\circ} \mathrm{C}\right)$. No changes in the shape (swelling) of the particles were observed at 20 , 60 , and $70 \%$ relative moisture content.

part of the epichlorohydrin is involved in cross-linkages. Furthermore, it must be noted that epichlorohydrin most likely does not form an ideal tetrafunctional network, as microheterogeneities can be present due to the formation of short free chain ends, loops, entanglements, and microaggregation of epichlorohydrin $(6,7)$. Experiments on bulk hydrogels indeed showed (article in preparation) that the cross-linking efficiency of Paselli SA-2 potato starch with epichlorohydrin was below 5\%. After $20 \mathrm{~s}$, the particles appear to be saturated with water (4e) and, subsequently, collapse on the grid (4f). Figure 5 shows the results of the same E-SEM experiments on samples 20Pa4.9E1O and $40 \mathrm{~Pa} 2 \mathrm{E} 1 \mathrm{O}$ differing in cross-linking degree. Almost no swelling or conformation change occurred upon raising of the moisture content. This clearly demonstrates the higher degree of crosslinking of 20Pa4.9E1O and 40Pa2E1O compared to 20Pa1E1O.

\subsection{Viscosity Measurements}

As the preparation of the starch microgels leads to the formation of colloids with a rather large size distribution, the light scattering method, in this case, was not suitable for determining accurately the particle size in suspension. For this reason, viscosity measurements were performed to obtain particle-specific volumes. In section 3.1, starch microgels were shown to be spherical, and assuming Eq. [8] is valid, the extrapolation of a linear fit of the reduced viscosity data to the zero particle concentration $\left(c_{\mathrm{p}} \rightarrow 0\right)$ should give the mass intrinsic viscosity ([ $\left.\left.\eta\right]\right)$. From $[\eta]$, the particle hydrodynamic volume is then deduced by employing Eq. [9]. However, for the starch colloidal suspensions at low ionic strength, $[\eta]$ cannot be determined from the extrapolation predicted by [8]. In Fig. 6, the specific viscosity and the reduced viscosity of deionized 20Pa1E1O suspensions are plotted as a function of $c_{\mathrm{p}}$. For $c_{\mathrm{p}}<1 \mathrm{w} \%$ (see Fig. 6a), the data can be fit by a linear regression, whereas the reduced viscosity reported in Fig. $6 \mathrm{~b}$ decreases on increasing $c_{\mathrm{p}}$. Hereafter, this decrease of the reduced viscosity with dilution will be referred to as an "anomalous" viscosity behavior. Due to this anomalous behavior, a linear fit of the reduced viscosity to $c_{\mathrm{p}} \rightarrow 0$ of the data presented in Fig. 6b is clearly not possible. A double logarithmic plot of the reduced viscosity versus the particle concentration presented in Fig. 6c shows a linear part, which is in fairly good agreement with an empirical scaling law proposed by Antonietti, for charged polystyrenesulfonate lattices $(17,30)$ :

$$
\eta_{\text {red }} \propto c_{\mathrm{p}}^{\alpha}
$$

According to experiments in (17), $\alpha$ is found to be -0.25 for spherical particles and $\sim-0.7$ for linear polyelectrolytes. The $\alpha$ values, reported in Table 4, suggest that the starch microgels behave more or less like spherical particles in aqueous suspensions. The larger $\alpha$ values obtained might be attributed to loose ends.

The anomalous viscosity behavior is also well described by Fuoss's empirical relation for semiflexible polyelectrolytes (14),

$$
\frac{\eta_{\mathrm{sp}}}{c_{\mathrm{p}}}=\frac{A}{1+B \sqrt{c_{\mathrm{p}}}}
$$

where, $A$ and $B$ are constants. Since starch microgels may swell, a fit of our data presented in Fig. 6d according to [12]

TABLE 4

Fit Results Obtained by Applying Antonietti's Scaling Law ( $\alpha$ ) and Fuoss's Relation for Semiflexible Polyelectrolytes $(A)$

\begin{tabular}{ccr}
\hline Starch microgel suspensions & $\alpha$ & $A$ \\
\hline 20Pa1E1OH & -0.39 & 333 \\
$20 \mathrm{~Pa} 2 \mathrm{E} 1 \mathrm{OH}$ & -0.23 & 45 \\
\hline
\end{tabular}




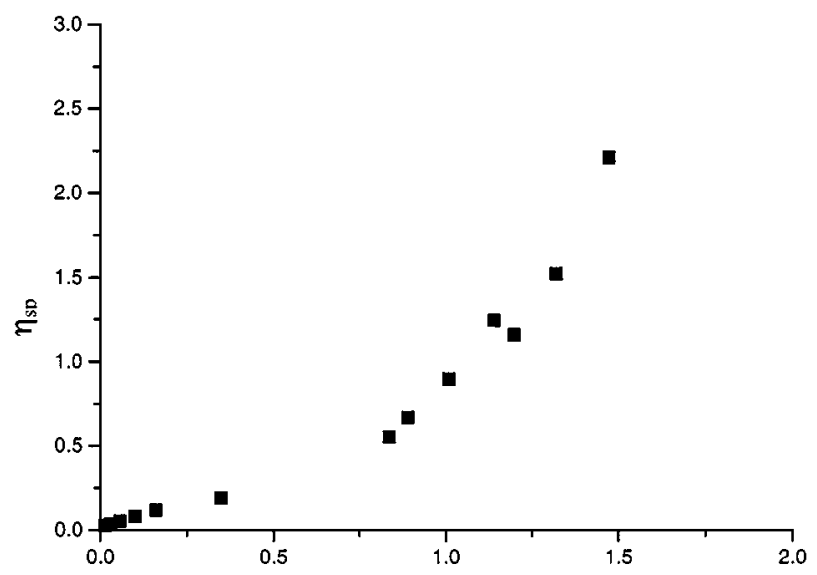

a)

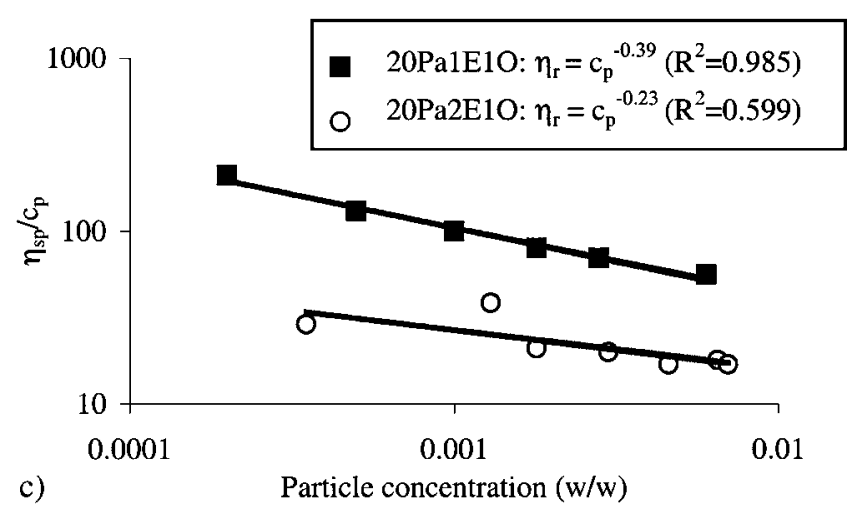

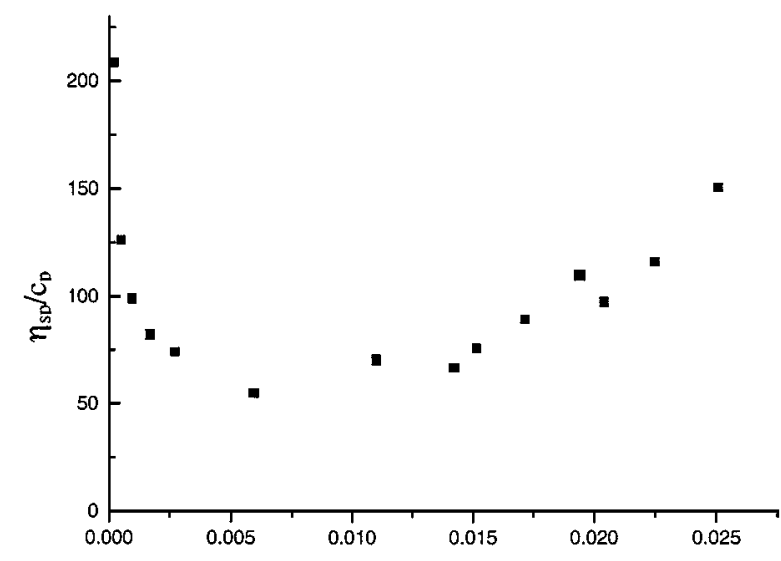

b)

Particle concentration (w/w)

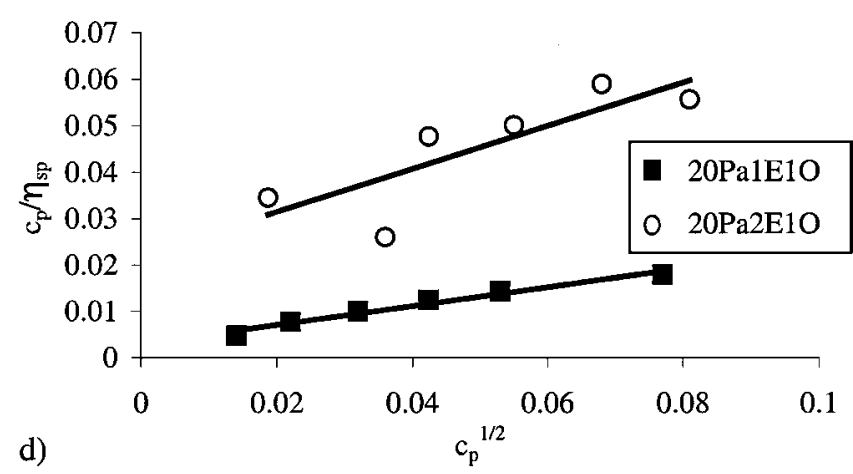

FIG. 6. (a) Specific viscosity of $20 \mathrm{~Pa} 1 \mathrm{E} 1 \mathrm{O}$ versus particle weight concentration $\left(c_{\mathrm{p}}\right)\left(20.0^{\circ} \mathrm{C}\right)$. For $c_{\mathrm{p}}<0.8 \%$, the specific viscosity increases linearly with the particle concentration. (b) $\eta_{\mathrm{sp}} / c_{\mathrm{p}}$ versus particle weight concentration $\left(c_{\mathrm{p}}\right)$ of $20 \mathrm{~Pa} 1 \mathrm{E} 1 \mathrm{O}\left(20.0^{\circ} \mathrm{C}\right)$. For $c_{\mathrm{p}}<0.8 \%$, the reduced viscosity decreases with dilution. (c) Double logarithmic plot of the reduced viscosity versus particle concentration of ( $\square$ ) 20Pa1E1O and (O) 20Pa2E1O suspensions. The linear part of the curve

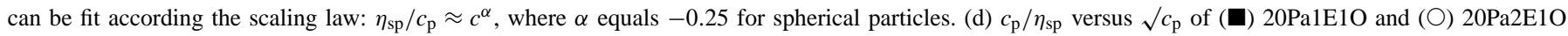
suspensions. The curve can be linearly fit according to Fuoss's empirical equation.

appears to be reasonable. If the extrapolation to $c \rightarrow 0$ is indeed justified, i.e., if the equation is applicable up to $c \rightarrow 0$, then $A$ represents the mass intrinsic viscosity. The $A$ values obtained for 20Pa1E1OH and 20Pa2E1OH (see Table 4) are much larger than expected for uncharged starch microgels (compare to, for example, [8]), which is probably due to surface charges.

In Fig. 7, the reduced viscosity of 20Pa2E1O for deionized suspensions $\left(\kappa_{\mathrm{S}} a=2\right)$ and for $1 \mathrm{M} \mathrm{NaCl}$ suspensions $\left(\kappa_{\mathrm{s}} a>100\right)$ is given as function of $c_{\mathrm{p}}$. In the case of $\kappa_{\mathrm{s}} a=2$, the anomalous viscosity behavior, persisting for $c_{\mathrm{p}}<0.8 \mathrm{w} \%$, disappears for higher particle concentrations. For $c_{\mathrm{p}}>0.8 \mathrm{w} \%$, the reduced viscosity increases linearly with the particle concentration. This trend is also observed by Antonietti for charged lattices $(17,28)$. The linear increase, in Fig. 7, can be attributed to an increasing importance of interactions. Since no agglomerates were observed (i.e., before and several weeks after the measurements the color of all the suspensions remained transparent blue), these particle interactions are not attractive. Although the increasing counterions concentration, produced by the starch microgels themselves, tend to screen the repulsions between the particle double layers, a net charge number per microgel apparently still favors these repulsions, being responsible for the increase of the reduced viscosity. For $c_{\mathrm{p}}>0.8 \mathrm{w} \%$, Eq. [3] is not appropriate anymore to calculate the particle double layer $\left(\kappa^{-1}\right)$ in suspension, as the concentration of counterions produced by the particles themselves becomes not negligible anymore. In this case, the Debye length $\left(\kappa^{-1}\right)$ must be calculated taking the contribution of the particle concentration into account as well (see [1]).

For the case $\kappa_{\mathrm{s}} a>100$ (see Fig. 7), the trend of the reduced viscosity increases linearly over the complete concentration range, as also observed for neutral polymers in good solvents. A linear fit of the data gives $[\eta]$ and $k_{\mathrm{H}}$, which are 6 and 60 , respectively. The rather low $[\eta]$ value approaches the hard sphere limit. However, the experimental Huggins coefficient $k_{\mathrm{H}}$ is approximately 100 times larger than the value predicted for hard spheres $\left(k_{\mathrm{H}}=0.64\right)$. In our case, for $c_{\mathrm{p}}>0.1 \mathrm{w} \%$, agglomerates, which 


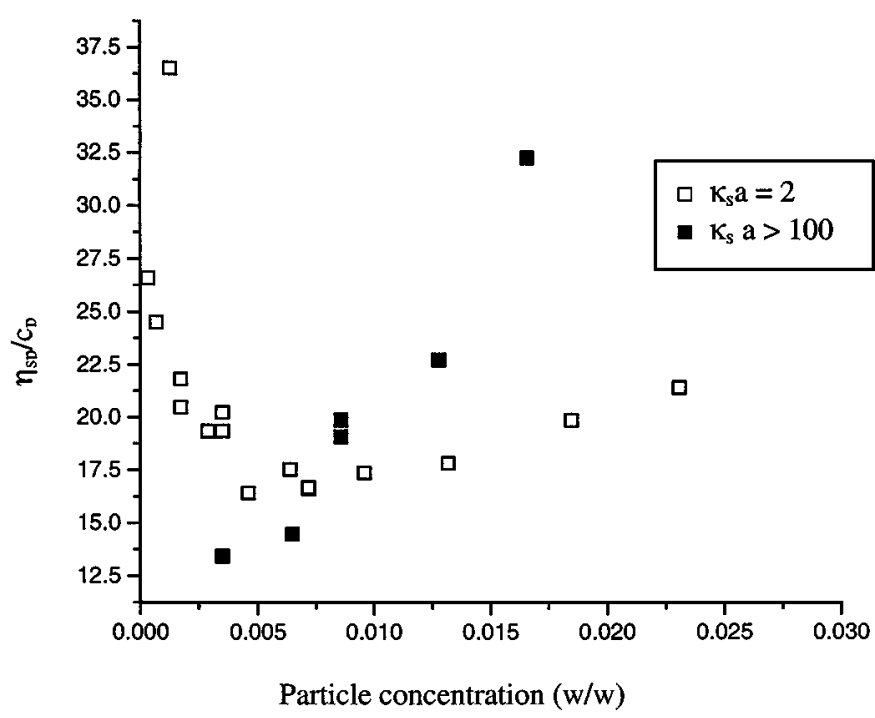

FIG. 7. $\eta_{\mathrm{sp}} / c_{\mathrm{p}}$ versus particle weight concentration $\left(c_{\mathrm{p}}\right)$ for $(\square)$ salt-free and for $(\boldsymbol{\square})[\mathrm{NaCl}]=1 \mathrm{M}$ suspensions of $20 \mathrm{~Pa} 2 \mathrm{E} 1 \mathrm{O}\left(20.00^{\circ} \mathrm{C}\right)$. The nonlinear dependence becomes linear at higher particle concentrations. At a much higher ionic strength, the microgels form aggregates.

appeared on the walls of the bottles subsequently after the experiments, suggest that van der Waals attractions are mainly responsible for the high Huggins coefficient (also found by Antonietti (17)).

Summarizing the observations made for Figs. $6 \mathrm{~b}$ and 7 , we conclude that, at very low ionic strengths, the starch microgels appear to be sufficiently stabilized by the dissociated hydroxyl groups of the glucose units, whereas at high ionic strength the effect of van der Waals attraction becomes noticeable.

E-SEM experiments have shown that the swelling of starch microgels depends on the cross-linking degree, even for low cross-linking degrees (see section 3.2). Rheological experiments on salt-free suspensions of starch microgels differing in crosslinking degree were performed to observe any effect of the cross-linking on the anomalous viscosity behavior, as already observed for polystyrenesulfonate lattices (17). In Fig. 8, the reduced viscosity of 20Pa1E1O and 20Pa2E1O is plotted versus $c_{\mathrm{p}}$, for similar $\kappa_{\mathrm{s}} a$. For both samples, a decrease of the reduced viscosity with increasing dilution is observed for $c_{\mathrm{p}}<1 \mathrm{w} \%$. However, the reduced viscosity values of $20 \mathrm{~Pa} 2 \mathrm{E} 1 \mathrm{O}$ are about four times lower than values of 20Pa1E1O. For $c_{\mathrm{p}}>1 \mathrm{w} \%$, the curves are linear for both samples, with still a higher reduced viscosity for 20Pa1E1O than for 20Pa2E1O. An increase of the cross-linking degree diminishes significantly the reduced viscosity of starch microgels in aqueous suspensions. However, the cross-linking degree seems to have no effect on the occurrence of the anomalous viscosity behavior at low particle concentrations. Apparently, this anomalous behavior is, for starch microgels, not related to any particle structural change in size or shape (i.e., swelling). Hence, the persistence of the anomalous viscosity behavior implies that swelling and effective charge number per microgel must be proportional, as also found by Antonietti for polystyrenesulfonate lattices (17). This proportionality suppresses the effect of particle size (see below) on the anomalous viscosity behavior.

In Figs. 6b, 7, and 8, we have shown that the anomalous reduced viscosity dependence also occurs for much larger particle sizes than those referred to in the literature (17). To determine if this trend is also observable for particle sizes even in the range of a micrometer, the reduced viscosity of sample 20Pa4T1OA (with a radius $(a)$ of $2.5 \mu \mathrm{m})$ and 20Pa4T1OB $(a=304 \mathrm{~nm})$ versus $c_{\mathrm{p}}$ is presented in Fig. 9. Both systems were deionized, and only differ in $\kappa_{\mathrm{s}} a$. For $\kappa_{\mathrm{s}} a=3.2$, the anomalous viscosity behavior is found again, whereas the trend is linear for $\kappa_{\mathrm{s}} a=26$, in the same weight concentration range. As a large particle radius contributes substantially to the increase of $\kappa_{\mathrm{s}} a$, then for micrometer particles, $\kappa_{\mathrm{s}} a$ is already too large to observe any anomalous viscosity behavior. The disappearance of the anomalous reduced viscosity behavior for particles larger than $1 \mu \mathrm{m}$ can be qualitatively explained as follows: small particles $(<1 \mu \mathrm{m})$ have a higher specific surface area and, consequently, a higher number of charges per gram. Thus, small submicrometer particles have a higher number of associated counterions. As electrostatic effects are more dominant for small particles than for larger ones, the increase of the intrinsic viscosity with particle dilution is more pronounced.

Experiments on gelatinized starch showed that $\mathrm{NaCl}$ could be used as a swelling inhibitor (25). A significant increase of the ionic strength leads to a decrease of the volume of the hydrogel, as a result of osmosis $(6,7)$. Similarly, an increase of the ionic strength may affect the swelling of starch microgels. In Fig. 10, the reduced viscosity of sample 20Pa4T1OA

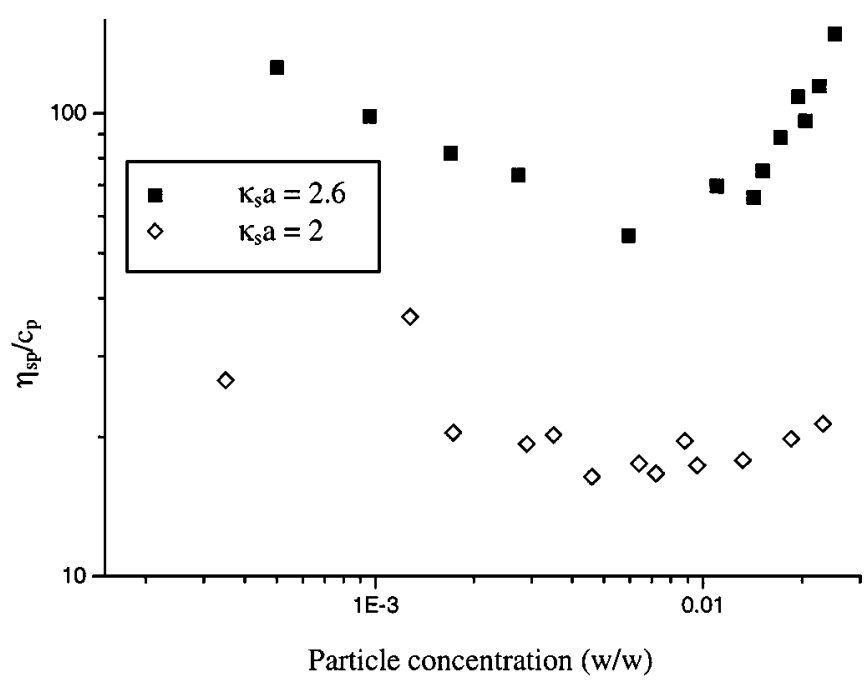

FIG. 8. Dependence of $\eta_{\mathrm{sp}} / c_{\mathrm{p}}$ on the cross-linking degree of starch-based hydrogels for $(\boldsymbol{\square}) 20 \mathrm{~Pa} 1 \mathrm{E} 1 \mathrm{O}$ and $(\diamond) 20 \mathrm{~Pa} 2 \mathrm{E} 1 \mathrm{O}$ suspensions (same $\kappa_{\mathrm{s}} a$ ) at $20.00^{\circ} \mathrm{C}$. 


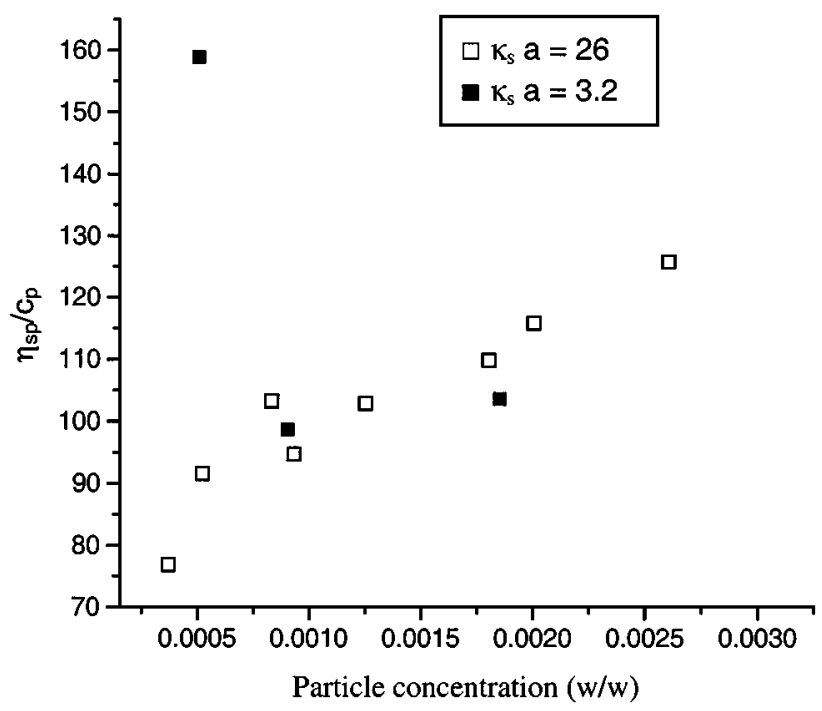

FIG. 9. Influence of the size of starch-based particles $\left(\kappa_{\mathrm{s}} a\right)$ on $\eta_{\mathrm{sp}} / c_{\mathrm{p}}$ of ( $\square$ ) 20Pa4T2OA and (ם) 20Pa4T2OB suspensions $\left(20.00^{\circ} \mathrm{C}\right)$.

is illustrated, for $\kappa_{\mathrm{s}} a$ is 26,250 , and 500. In all cases within the range of measured concentrations, the trend of the reduced viscosity is increasing linearly. An extrapolation to zero concentration gives $[\eta]$ equal to $79.6,16$, and 14.3 respectively, showing that with increasing ionic strength the starch colloids are approaching the noninteracting sphere limit. Although for $\kappa_{\mathrm{s}} a=250$ and $\kappa_{\mathrm{s}} a=500$, the reduced viscosity is strongly reduced by five times compared to $\kappa_{\mathrm{S}} a=26$, changes in the particle size were not observable by static light scattering. In Table $5, k_{\mathrm{H}}$ values are reported for the different systems. The

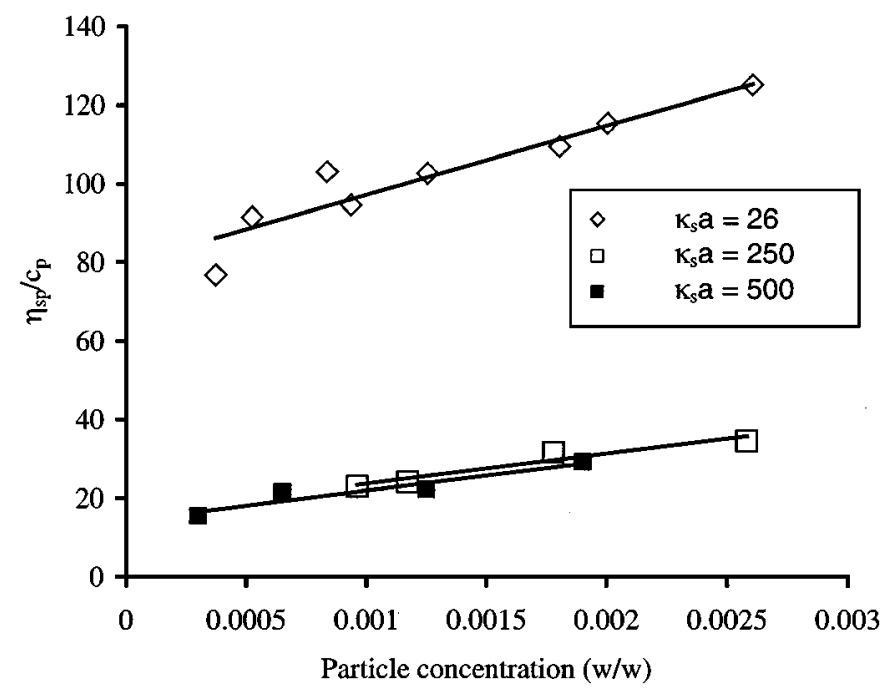

FIG. 10. Influence of the ionic strength $\left(\kappa_{\mathrm{s}} a\right)$ on $\eta_{\mathrm{sp}} / c_{\mathrm{p}}$ for $(\diamond)$ deionized, 口) $[\mathrm{NaCl}]=0.0034 \mathrm{M}$, and $(\square)[\mathrm{NaCl}]=0.015 \mathrm{M}$ suspensions of $20 \mathrm{~Pa} 4 \mathrm{~T} 2 \mathrm{OA}$ $\left(20.00^{\circ} \mathrm{C}\right)$.
TABLE 5

$k_{\mathrm{H}}$ Values in Function of $\kappa_{\mathrm{s}} a$ for Sample 20Pa4T1OA

\begin{tabular}{rl}
\hline$\kappa_{\mathrm{S}} a$ & $k_{\mathrm{H}}$ \\
\hline 26 & 2.8 \\
250 & 30.2 \\
500 & 38 \\
\hline
\end{tabular}

increase of $k_{\mathrm{H}}$ values for increasing $\kappa_{\mathrm{s}} a$ and the absence of aggregates in the suspensions indicate that particle interactions are still repulsive (see remarks for Fig. 7). An increase of the ionic strength apparently screens mostly the charges at the surface of starch microgels instead of the internal charges, as it was also concluded in Antonietti's work (17). This is of importance, as until now the behavior of starch microgels in aqueous suspensions was assumed to be directly related to the properties of macroscopic starch cross-linked hydrogels. Apparently, the osmotic driving force strongly influencing the swelling of macro-hydrogels in aqueous suspensions is not dominant anymore, compared to the influence of the ionic strength on the Debye length, in the behavior of colloidal microgel suspensions.

\subsection{Charge-Stabilized Silica Spheres in Ethanol}

This work showed that the decrease of the reduced viscosity on increasing the particle concentration also occurs for the complex structured starch microgels. In the anomalous reduced viscosity behavior, which still persists on increasing the crosslinking degree of the starch microgels, the $\kappa_{\mathrm{s}} a$ values seem to be the essential parameter. Conformation changes (or particle swelling) are not solely responsible for the anomalous viscosity behavior, as is claimed for semiflexible polyelectrolytes (1416). It may be a general feature for charged colloids, irrespective of their rigidity. In fact, the results for starch colloids show that a decrease of the reduced viscosity at increasing particle concentration only occurs for low $\kappa_{\mathrm{s}} a$ values (or $\kappa a$ ). To determine whether a low $\kappa_{\mathrm{s}} a$ is indeed sufficient to produce this phenomenon, the viscosity results of the silica spheres for decreasing values of $\kappa_{\mathrm{s}} a$ are presented in Fig. 11. Figure 11a presents values obtained for deionized particles, where the reduced viscosity decreases with increasing particle concentration. Note the comparable values obtained for DB373 rigid silica spheres (Fig. 11a) and for 20Pa1E1OH starch colloids (Fig. 6b), at similar $\kappa_{\mathrm{s}} a$. In Figs. 11b-d, the anomalous viscosity behavior levels out at higher salt concentrations, approaching the hard sphere limit. Clearly, charged rigid spheres in deionized non aqueous suspensions also show the anomalous viscosity behavior, which in the case of TPM-silica spheres cannot be due to conformation changes. The only relevant parameter affecting the high reduced viscosity values of dilute suspensions of silica spheres is manifestly the Debye length $\left(\kappa^{-1}\right)$. 


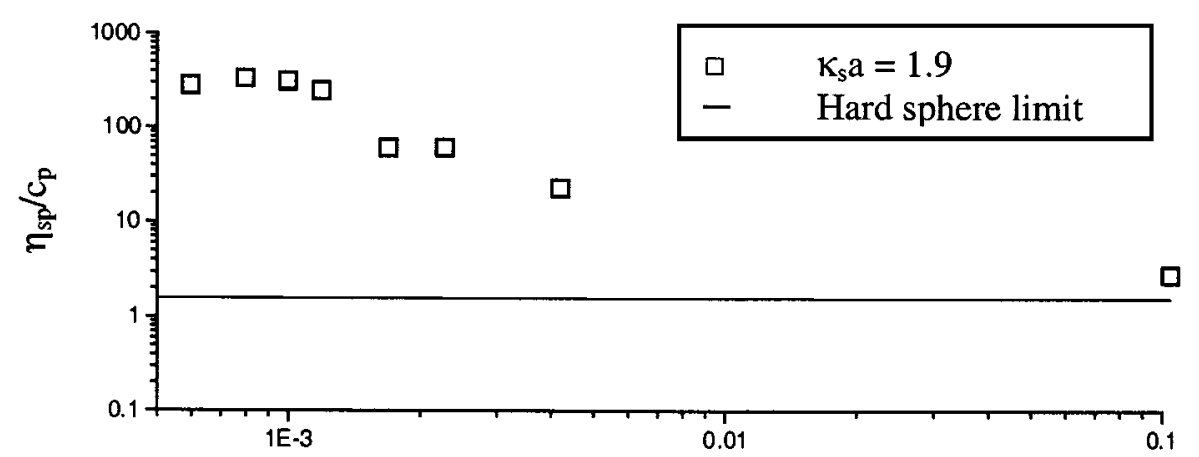

a)

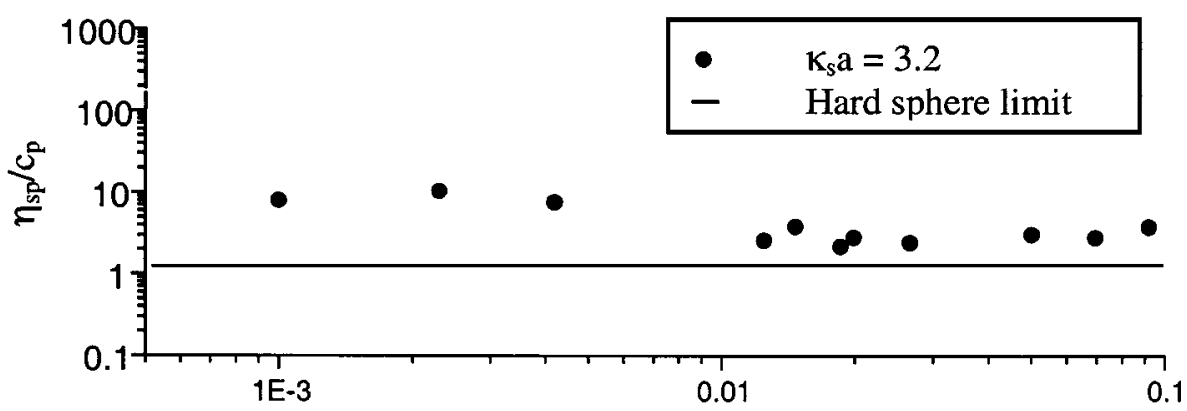

b)

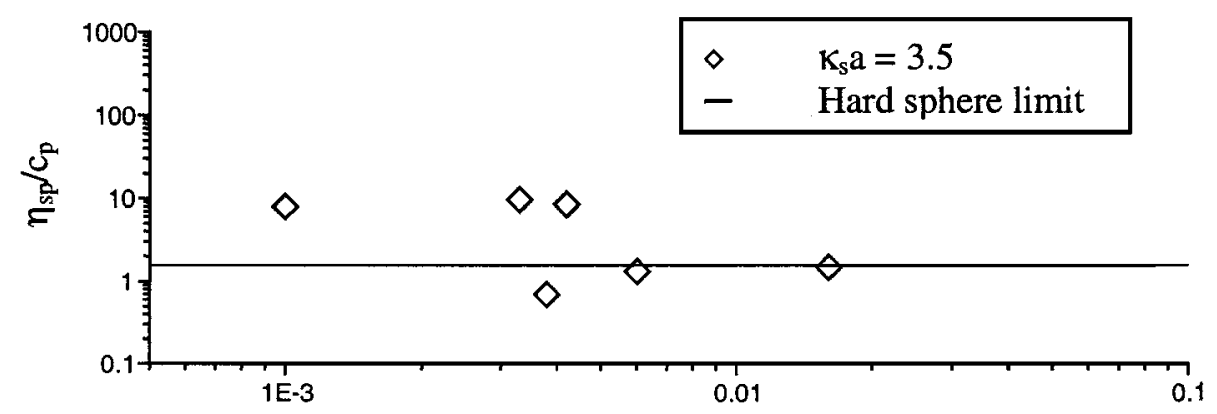

c)

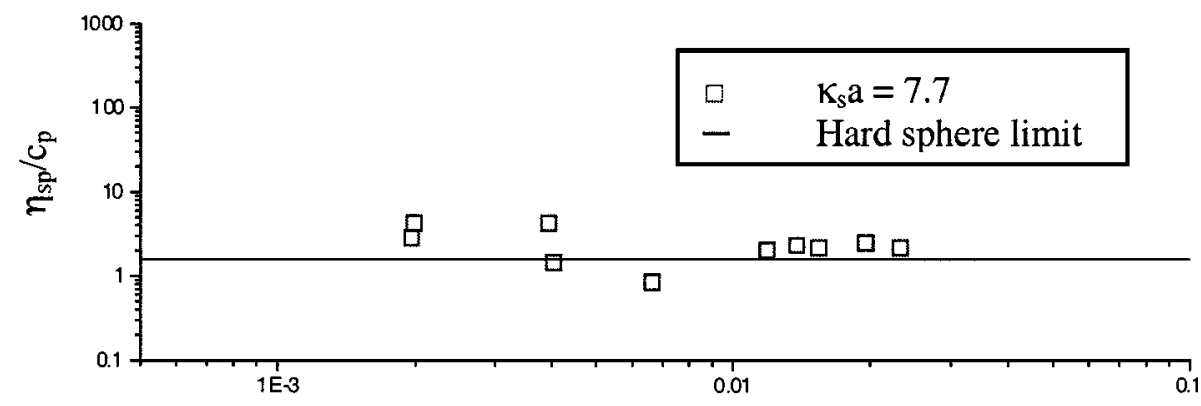

d)

Particle concentration (w/w)

FIG. 11. $\eta_{\mathrm{sp}} / c_{\mathrm{p}}$ versus particle weight concentration $\left(c_{\mathrm{p}}\right)$ of charge-stabilized TPM-silica spheres in ethanol for different ionic strengths (from $\kappa_{\mathrm{S}} a=1.9$ up to $\left.\kappa_{\mathrm{S}} a=7.7\right)$, at $20.00^{\circ} \mathrm{C}$. For $c_{\mathrm{p}}<0.1 \%$, the reduced viscosity decreases with increasing $\kappa_{s} a$, approaching the hard sphere limit. 


\section{CONCLUSIONS}

The present work reports a reproducible procedure for the preparation of novel starch microgels. From various characterization methods, it has been shown that the negatively charged microgels are spherical and amorphous, with sizes in the submicrometer range. The particle size strongly depended on the type of cross-links ((poly)phosphate or glycerol-like groups) introduced during the synthesis. E-SEM and viscosimetry clearly showed that the swelling properties of the starch colloids are strongly affected by the increase of the cross-linking degree of the microgel. The usual determination of the specific volume of starch colloids in salt-free aqueous suspensions via the intrinsic viscosity showed that the complex structured starch microgels behave like charged semiflexible polymers, as the reduced viscosity increases with particle dilution. This anomalous viscosity behavior also occurs for other colloids, namely charged rigid spheres, provided the ionic strength is sufficiently low. Changes in shape of the polymers appear to play a minor role in the anomalous viscosity behavior, which is more likely dominated by electrostatic effects.

Starch colloids present, at first sight, suitable properties (size, charge, density) to be used as thickeners in applications like paints. Further work on the rheological behavior of the spherical starch microgels in higher concentrated regimes will be performed, as applications involving starch colloids also require more detailed information on the behavior of the microgels in aqueous suspension.

\section{ACKNOWLEDGMENTS}

P. van Maurik and FEI (Eindhoven, The Netherlands) for making E-SEM pictures of the starch-based microgels are gratefully acknowledged. We thank J. Wijnhoven for the SEM and TEM pictures. This work is supported by the Marie Curie Grant ERBFAIRCT985014.

\section{REFERENCES}

1. Ellis, R. P., Cochrane, M. P., Dale, M. F. B., Duffus, C. M., Lynn, A., Morrison, I. M., Prentice, R. D. M., Swanston, J. S., and Tiller, S. A., J. Sci. Food Agric. 77, 289 (1998).
2. Jane, J., J. Macromol. Sci. 4 A32, 751 (1995).

3. Soest, J. J. G. van, Schijndel, R. J. G. van, Gotlieb, K. F. P., and Stappers, F. J. M., PCT WO 40617 (2000).

4. Soest, J. J. G. van, Verduin, H., and Feil, H., "Second World Congress on Emulsion (Conference Book).” EDS Editeur, Paris, 1997.

5. Jiugao, Y., and Jie, L., Starch 46, 252 (1994).

6. Kulicke, W.-M., Aggour, Y. A., Nottelmann, H., and Elsabee, M. Z., Starch 41, 140 (1989).

7. Kulicke, W.-M., Aggour, Y. A., and Elsabee, M. Z., Starch 42, 134 (1990).

8. Kartha, K. P. R., and Srivasta, H. C., Starch 37, 291 (1985).

9. Dickinson, E., "An Introduction to Food Colloids." Oxford Sci., Oxford, 1992.

10. Hunter, R. J., "Foundations of Colloid Science," Vol. I. Clarendon, Oxford 1986.

11. Makosko, C. W., "Rheology, Principles, Measurements and Applications." VCH, New York, 1994.

12. Mancuso, J. F., Maxwell, W. B., and Danilatos, G. D., US patent application US4880976 (1989).

13. Philips Electron Optics, The Netherlands, ISBN 90-907755-3.

14. Füoss, R. M., and Strauss, U. P., J. Polym. Sci. 3, 602 (1948).

15. Flory, P. J., "Principles of Polymer Science." Cornell Univ. Press, Ithaca, NY, 1953.

16. Mysels, K. J., "Introduction to Colloid Chemistry." Wiley, New York, 1967.

17. Antonietti, M., Briel, A., and Förster, S., J. Chem. Phys. 105, 7795 (1996).

18. Carrington, S. P., Fisher, L. R., and Odell, J. A., "Starch Structure and Functionality." R. Soc. Chem., Cambridge, 1997.

19. Philipse, A. P., and Vrij, A., J. Colloid Interface Sci. 28, 121 (1989).

20. Beresford-Smith, B., Chan, D. Y. C., and Mitchell, D. J., J. Colloid Interface Sci. 105, 216 (1985).

21. Philipse, A. P., and Vrij, A., J. Chem. Phys. 88, 6459 (1989).

22. Soest, J. J. G. van, Tournois, H., Wit, D. de, and Vliegenthart, J. F. G., Carbohydr. Res. 279, 201 (1995).

23. Hirs, C. H. W., and Timashejf, S. N., Methods Enzymol. 61 (1979).

24. Walstra, P., "Encyclopedia of Emulsion Technology" (P. Becher, Eds.), Vol. 1. Dekker, New York, 1983.

25. Oosten, B. J., Starch 42, 327 (1990).

26. Lide, D. R. (Ed.), "Handbook of Chemistry and Physics," 78th ed. CRC Press, Boca Raton, FL, 1997.

27. Rijke, A. M., Ph.D. thesis, Leiden Univ., The Netherlands, 1961.

28. Holmberg, L., Lindberg, B., and Lindqvist, B., Carbohydr. Res. 268, 47 (1995).

29. Holmberg, L., Lindberg, B., and Lindqvist, B., Carbohydr. Res. 272, 203 (1995).

30. Antonietti, M., Briel, A., and Förster, S., Macromolecules 30, 2700 (1997). 